\title{
Changes of the spin dynamics in perovskite and bilayer manganite
}

\author{
$\underline{\text { A. I. Coldea }}^{\text {a }}$, S. J. Blundell ${ }^{\text {a }}$, C. A. Steer ${ }^{\text {a }}$, F. L. Pratt ${ }^{b}$, \\ D. Prabhakaran ${ }^{\mathrm{a}}$, J. F. Mitchell ${ }^{\mathrm{c}}$ \\ ${ }^{a}$ Department of Physics, University of Oxford, Parks Road, Oxford, OX1 3PU, \\ United Kingdom \\ b Rutherford Appleton Laboratory, Chilton, Didcot, OX11 0QX, United Kingdom \\ ${ }^{\mathrm{c}}$ Materials Science Division, Argonne National Laboratory, Argonne, Illinois \\ 60439, USA
}

\begin{abstract}
We report a series of $\mu \mathrm{SR}$ measurements performed on single crystals of $\mathrm{La}_{1-x} \mathrm{Sr}_{x} \mathrm{MnO}_{3}$ $(0 \leq x \leq 0.125)$ and on a polycrystalline bilayer manganite $\mathrm{La}_{2-2 x} \mathrm{Sr}_{1+2 x} \mathrm{Mn}_{2} \mathrm{O}_{7}$ $(x=0.52)$. We find that the temperature dependence of the spin-lattice relaxation rate is strongly dependent on the hole doping and the associated changes in the magnetic structure. The systems have competing, anisotropic interactions, leading to a complex interplay of charge, spin and orbital order. This has a significant influence on the spin dynamics, both for perovskite and layered manganites.
\end{abstract}

Key words: Muon spin relaxation, manganites, spin dynamics

Corresponding author:

A. I. Coldea, Oxford University Department of Physics, Clarendon Laboratory, Parks Road, Oxford OX1 3PU, United Kingdom. Tel. +44 1865 272370, FAX: 
+44 1865 272400, email: a.coldea@physics.ox.ac.uk

The rich phase diagram of the colossal magnetoresistive (CMR) manganites is determined by the coexistence of different types of interactions on comparable energy scales which produces a complex interplay of magnetism, electronlattice coupling and orbital and charge ordering. The phase separation into ferromagnetic (FM), metallic and insulating antiferromagnetic (AFM) regions has been suggested as a possible reason for the observation of the CMR effect [1]. In this paper we present a muon spin relaxation $(\mu \mathrm{SR})$ study on lowdoped perovskite manganites investigating the changes in the spin dynamics by varying the hole doping of single crystals of $\mathrm{La}_{1-x} \mathrm{Sr}_{x} \mathrm{MnO}_{3}(0 \leq x<0.125)$. The canted antiferromagnetic structure observed in this part of the phase diagram has been often explained in terms of microscopic phase separation into hole rich ferromagnetic and less doped antiferromagnetic regions $[2,3]$. We find a significant increase in the relaxation rate for a sample with $x=0.10$, which is consistent with the presence of different magnetic phases. A phase separation into a charge-ordered (CO) and an A-type AFM phase is also found in the bilayer compound $\mathrm{La}_{2-2 x} \mathrm{Sr}_{1+2 x} \mathrm{Mn}_{2} \mathrm{O}_{7}$ with $x=0.52$.

Zero-field $\mu \mathrm{SR}$ is especially suited for study of short-range magnetic correlations and dynamics of the spin fluctuations since the positive muons are a sensitive local probe. Because the local magnetic fields at muon sites result primarily from dipolar interactions which decay very quickly with increasing distance $\left(\sim 1 / r^{3}\right)$ the effective range investigated with muons is $\sim 20 \AA$. Single crystalline samples of $\mathrm{La}_{1-x} \mathrm{Sr}_{x} \mathrm{MnO}_{3}$ were prepared using a floating zone image furnace whereas polycrystalline samples $(\sim 0.5 \mathrm{~g})$ were prepared as described in Ref. [4]. Zero-field $\mu \mathrm{SR}$ data were taken on the EMU beamline at the ISIS facility (Rutherford Appleton Laboratory). The ISIS data do not show any oscillations (whose frequencies are proportional to the local magnetic fields) or fast relaxing components, due to the limited frequency and relaxation window. 
The changes in the magnetic structure as a function of hole doping were investigated using $a c$ susceptibility. The temperature dependence of the real part of the susceptibility, $\chi^{\prime}$, of the single crystals of the $\mathrm{La}_{1-x} \mathrm{Sr}_{x} \mathrm{MnO}_{3}$ compounds is shown in Figure 1. The parent compound, $\mathrm{LaMnO}_{3}$, shows a very sharp peak at $T=136.2 \mathrm{~K}$ and a small feature around $T^{*}=106 \mathrm{~K}$. The large peak is close to the transition temperature of the A-type AFM structure [5], as found in neutron diffraction measurements $\left(T_{\mathrm{N}}=139.5 \mathrm{~K}\right)[5]$. However, the large narrow peak in the $a c$ susceptibility is uncommon for a classical antiferromagnet where the susceptibility varies according to a Curie-Weiss law above the transition temperature. The ideal A-type AFM structure for $x=0$ consists of $(a b)$ ferromagnetic planes coupled antiferromagnetically along the $c$ axis. However, the canting of the magnetic structure (weak ferromagnetic moment along the $c$ axis) even for stoichiometric $x=0$ has been reported and it may originate from the interplay between the in-plane antisymmetric Dzyaloshinsky-Moriya interaction, single ion anisotropy [6], and lattice distortion [7]. This spin canting could be the origin of the large increase in susceptibility at $T_{\mathrm{N}}$ while the second peak at $T^{*}$ could be due to a deviation from perfect stoichiometry [6].

By introducing holes into $\mathrm{LaMnO}_{3}$ one creates double-exchange interactions [8] that compete with the superexchange interactions and for low hole doping either a canted antiferromagnetic structure (small ferromagnetic magnetic moment along $c$ ) is stabilized or a phase separation into ferromagnetic and antiferromagnetic regions occurs [9]. The susceptibility for $x=0.05$, shows features similar to those obtained for $x=0$, with a large peak at $T_{\mathrm{N}}=130 \mathrm{~K}$ and a smaller peak at $T^{*}=117 \mathrm{~K}$ (Figure 1 ). For higher hole concentrations ferromagnetism occurs and the magnetic interactions become more isotropic. The susceptibility data for $x=0.10$ still show two peaks, (at $136 \mathrm{~K}$ and $118 \mathrm{~K}$ ) of similar intensities and much broader than the ones observed for $x=0$ and $x=0.05$ (Figure 1). Two well-defined magnetic transitions at $T_{\mathrm{CO}}=150 \mathrm{~K}$ (the sharp discontinuity in susceptibility) and $T_{\mathrm{C}}=171 \mathrm{~K}$ are identified for $x=0.125$ 
(Figure 1). The transition at $T_{\mathrm{CO}}$ is a first order transition and is produced by charge ordering [10].

The $\mu$ SR data collected at ISIS mainly provide information about the relaxing component which is fitted using a single exponential function, $G_{z}(t)=$ $A_{0} \exp (-\lambda t)$, where $A_{0}$ is the initial asymmetry and $\lambda$ is the relaxation rate. The fitting parameters, relaxation rate $\lambda$ and the initial asymmetry $A_{0}$, are presented in Figure 2. The asymmetry changes as the systems go through their magnetic transition, from $A_{0}$ (in the paramagnetic region) to $A_{0} / 3$ (in the magnetic region). This suggests that only the component of muon polarization parallel to the local magnetization is preserved in the ordered region. The asymmetry for $x=0$ drops from $A_{0}$ to $A_{0} / 3$ at $T_{\mathrm{N}}=138 \mathrm{~K}$ whereas for the other compositions the transitions are $T_{\mathrm{N}}=135 \mathrm{~K}$ for $x=0.05, T_{\mathrm{C}}=144 \mathrm{~K}$ for $x=0.10$ and $T_{\mathrm{C}}=176 \mathrm{~K}$ for $x=0.125$. The transition is very sharp for $x=0$ and $x=0.05$ and the asymmetry remains almost constant below $T_{\mathrm{N}}$ at about $A_{0} / 3$. On the other hand, samples with $x=0.10$ and $x=0.125$ have a broader transition region and the asymmetry at low temperature increases above $A_{0} / 3$ below $T_{\mathrm{C}}$. A similar tendency has been observed for a polycrystalline material with $x=0.125$, where the asymmetry increases in the magnetic region at temperatures lower than $T_{\mathrm{CO}}[11]$. The broad temperature range over which the local ordering develops, (seen in the asymmetry variation for $x=0.10$ and $x=0.125$ ), suggests that the samples are magnetically inhomogeneous around the transition temperature. Here magnetic clusters may compete with a particular type of Jahn-Teller distorted (insulating) phase [11].

The relaxation rate, $\lambda$, increases as the system approaches the magnetic transition from high temperatures due to the critical slowing down of the magnetic fluctuations. A maximum in $\lambda$ usually indicates the position of the transition temperature. An exception is $x=0.10$, for which the position of the relaxation maximum is at $T_{\mathrm{CO}}=125 \mathrm{~K}$, lower than the temperature for the drop in asymmetry at $A_{0} / 3\left(T_{\mathrm{C}}=140 \mathrm{~K}\right)$; this suggests that the magnetic transition takes 
place at $T_{\mathrm{C}}$ but the slowing down of the magnetic fluctuations happens only below $T_{\mathrm{CO}}$. This composition, $x=0.10$, also shows a very large relaxation rate in contrast with the other compositions. If data are analyzed assuming that the relaxation function is a stretched exponential function, $G_{z}(t)=A_{0} \exp (-\lambda t)^{\beta}$, the obtained values of $\beta$ are very small $(\beta<0.2)[12]$. This result is often used as an indication of two types of Mn-ion fluctuations [13]. Attempts to extract the two types of fluctuation over a limited temperature region give a slow component that has a maximum around $134 \mathrm{~K}$, and a fast component that behaves similarly to the relaxation rate, $\lambda$, already shown in Figure 2 . The possible presence of two types of relaxation are consistent with an inhomogeneous magnetic state between $T_{\mathrm{C}}$ and $T_{\mathrm{CO}}$, possible composed of ferromagnetic metallic and charge-ordered insulating regions.

We now demonstrate that phase separation also occurs in a related bilayer manganite $\mathrm{La}_{2-2 x} \mathrm{Sr}_{1+2 x} \mathrm{Mn}_{2} \mathrm{O}_{7}$ with $x=0.52$, but that the $\mu \mathrm{SR}$ data are dramatically different. The magnetization shown in Figure 3(a) is quite complex and it has an A-type AF transition at $T_{\mathrm{N}}=200 \mathrm{~K}$ and a charge ordering at $T_{\mathrm{CO}}=250 \mathrm{~K}$. This behavior is different from that expected for an A-type AF system, such as $\mathrm{LaMnO}_{3}$ (see Figure 1). The temperature dependence of the asymmetry is almost constant at $A_{0}$ for $T>T_{\mathrm{N}}$ but drops to about $2 A_{0} / 3$ below $T_{\mathrm{N}}$ (see Figure $3(\mathrm{~b})$ ). This suggests that only about $50 \%$ of the sample becomes magnetic, in contrast to $\mathrm{LaMnO}_{3}$ (see Figure 2), where the asymmetry reaches $\sim A_{0} / 3$ below $T_{\mathrm{N}}$. On further cooling below $T_{\mathrm{N}}$, the asymmetry for the bilayer compound decreases almost linearly with temperature and reaches $\sim A_{0} / 3$ for $T$ less than about $100 \mathrm{~K}$, suggesting that only at low temperatures is the whole volume of the polycrystalline material magnetically ordered. Thus, the variation of $A_{0}$ for $100 \mathrm{~K}<T<T_{\mathrm{N}}$ is consistent with the coexistence of $\mathrm{CO}$ and A-type AF phases. For $T<100 \mathrm{~K}$ the whole sample is ordered (either the A-type AF fraction dominates or the CO fractions transform into magnetic CE-type phase), in agreement with the discontinuity observed in the 
temperature dependence of the internal field at $100 \mathrm{~K}[12]$.

The increase in the relaxation rate, $\lambda$, due to the slowing down of magnetic spin fluctuations is observed above $T_{\mathrm{N}}$. However, in the case of the bilayer compound $\lambda$ increases even below $T_{\mathrm{N}}$ or remains very large (see Figure 3(c)), in contrast to perovskite materials (see Figure 2). The increase in $\lambda$ below $T_{\mathrm{N}}$ may be related to the enhanced magnetic fluctuations in bilayer manganites compared to perovskites due to the reduction in dimensionality. The relaxation rate, $\lambda$, has a broad peak centered around $50 \mathrm{~K}$ and the small change around $100 \mathrm{~K}$ can be correlated with the discontinuity of the internal field and changes in its distribution [12]. The CO disappears below $100 \mathrm{~K}$ and possibly reappears below $50 \mathrm{~K}$, as in the case of $x=0.5$ [14], and the broad maximum around $50 \mathrm{~K}$ in the relaxation rate of $\lambda$ is related to changes in the spin dynamics.

In conclusion, $\mu \mathrm{SR}$ provides evidence for phase separation into an A-type AF phase and a $\mathrm{CO}$ phase for $\mathrm{La}_{2-2 x} \mathrm{Sr}_{1+2 x} \mathrm{Mn}_{2} \mathrm{O}_{7}$ with $x=0.52$, similar to $x=0.50[14,15]$. Muon spin relaxation data on $\mathrm{La}_{1-x} \mathrm{Sr}_{x} \mathrm{MnO}_{3}$ with $x=0.10$ support the phase separation model in the $T_{\mathrm{CO}}<T<T_{\mathrm{C}}$ region due to increased dynamic fluctuations at $T_{\mathrm{CO}}$. In the bilayer manganite compound the magnetic fluctuations appear to be significantly enhanced compared with the perovskite materials.

\section{References}

[1] E. Dagotto, T. Hotta, A. Moreo, Physics Reports 244 (2001) 1.

[2] S. Uhlenbruck, R. Teipen, R. Klingeler, B. Bchner, O. Friedt, M. Hcker, H. Kierspel, T. Niemller, L. Pinsard, A. Revcolevschi, R. Gross, Phys. Rev. Lett. 82 (1999) 185.

[3] M. Paraskevopoulos, F. Mayr, J. Hemberger, A. Loidl, R. Heichele, D. Muller, A. A. Mukin, A. M. Balbashov, J. Phys.: Cond. Matter 12 (2000) 3993. 
[4] J. E. Millburn, J. F. Mitchell, D. N. Argyriou, Chem. Comm. 15 (1999) 1389.

[5] F. Moussa, M. Hennion, J. Rodriguez-Carvajal, A. H. Moudden, L. Pisard, A. Revcolevschi, Phys. Rev. B 54 (1996) 15149.

[6] V. Skumryev, F. Ott, J. M. D. Coey, A. Anane, J.-P. Renard, L. PinsardGaudart, A. Revcolevschi, Eur. Phys. J. B 11 (1999) 401.

[7] I. Solovyev, N. Hamada, K. Terakura, Phys. Rev. Lett. 76 (1996) 4825.

[8] P. G. de Gennes, Phys. Rev. B 118 (1960) 141.

[9] A. V. Korolyov, V. Y. Arkhipov, V. S. Gaviko, Y. Mukovskii, A. A. Arsenov, T. P. Lapina, S. D. Bader, J. S. Jiang, V. I. Nizhankovskii, J. Mag. Mag. Mater. $213(2000) 63$.

[10] H. Kawano, R. Kajimoto, M. Kubota, H. Yoshizawa, Phys. Rev. B 53 (1996) 14709.

[11] A. Llobet, J. L. Garcia-Muñoz, C. Frontera, M. Respaud, L. Pinsard, A. Revcolevschi, C. Scott, Physica B 289 (2000) 77.

[12] A. I. Coldea, Ph.D. thesis, Oxford University (2001).

[13] R. H. Heffner, J. E. Sonier, D. E. MacLaughlin, G. J. Nieuwenhuys, G. Ehlers, F. Mezei, S.-W. Cheong, J. S. Gardner, H. Roder, Phys. Rev. Lett. 85 (2000) 3285 .

[14] T. Chatterji, G. J. McIntyre, W. Caliebe, R. Suryanarayanan, G. Dhalenne, A. Revcolevschi, Phys. Rev. B 61 (2000) 570.

[15] D. N. Argyriou, H. N. B. B. J. Campbell, A. K. Cheetham, D. E. Cox, J. S. Gardner, K. Hanif, A. dos Santos, G. F. Strouse, Phys. Rev. B 61 (2000) 15269. 


\section{Figure Captions}

Fig. 1: Temperature dependence of the real part of the ac susceptibility measured at $10 \mathrm{kHz}$ for $\mathrm{La}_{1-x} \mathrm{Sr}_{x} \mathrm{MnO}_{3}$. The arrows indicate the position of different transition temperatures: Néel temperature, $T_{\mathrm{N}}$, Curie temperature, $T_{\mathrm{C}}$, charge-ordering temperature, $T_{\mathrm{CO}} . T^{*}$ is the temperature associated with the second peak in the susceptibility of crystals with $x \leq 0.10$.

Fig. 2: Temperature dependence of the initial asymmetry (left graph) and relaxation rate, $\lambda$, (right graph) for $\mathrm{La}_{1-x} \mathrm{Sr}_{x} \mathrm{MnO}_{3}$ with $x \leq 0.125$. The arrows indicate the positions of different transition temperatures.

Fig. 3: (a) Temperature dependence of zero field-cooled (ZFC) magnetization in a field of $10 \mathrm{mT}$, (b) the asymmetry and (c) the spin-lattice relaxation, $\lambda$, for $\mathrm{La}_{2-2 x} \mathrm{Sr}_{1+2 x} \mathrm{Mn}_{2} \mathrm{O}_{7}$ with $x=0.52$. The arrows indicate different transition temperatures. 


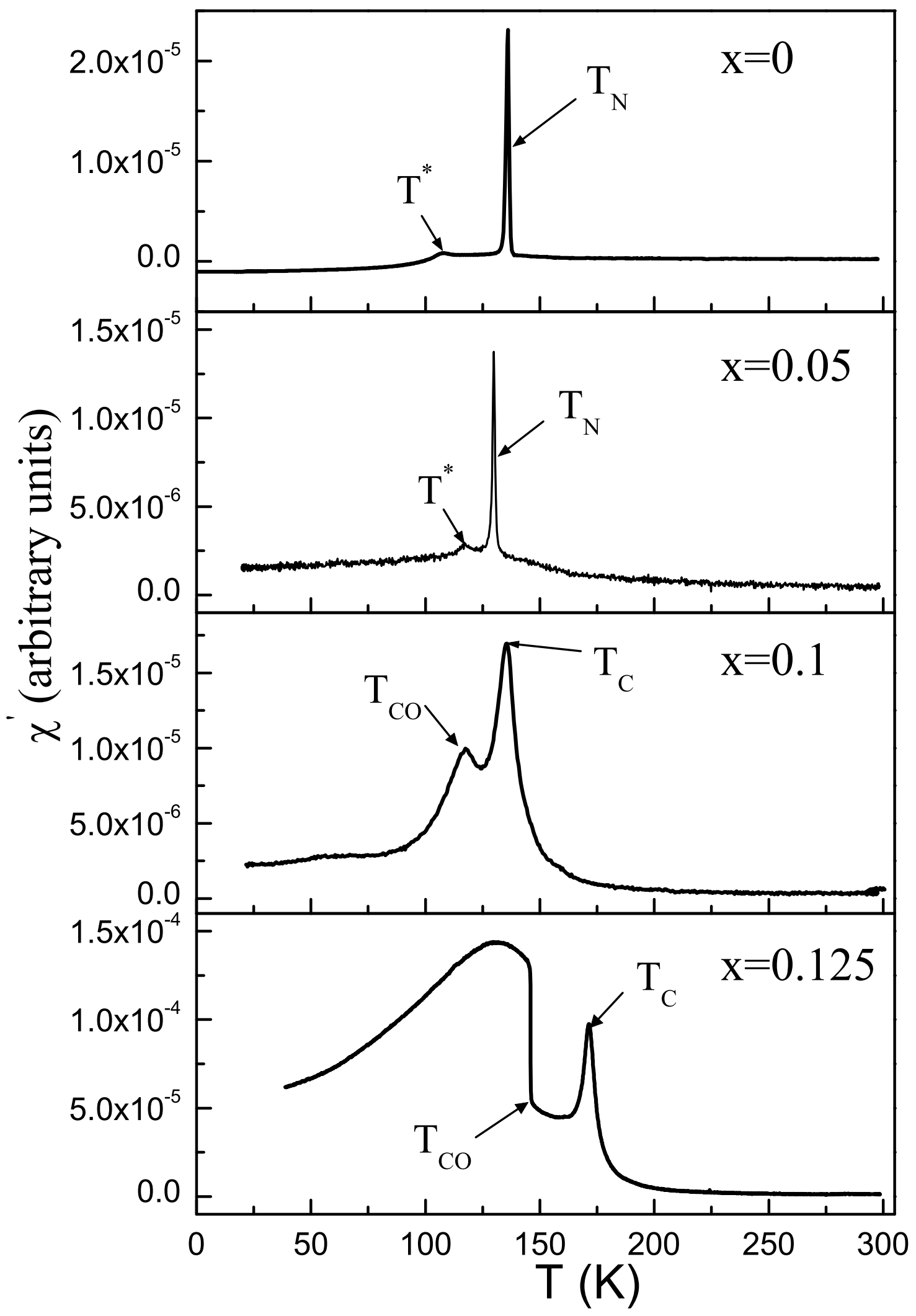



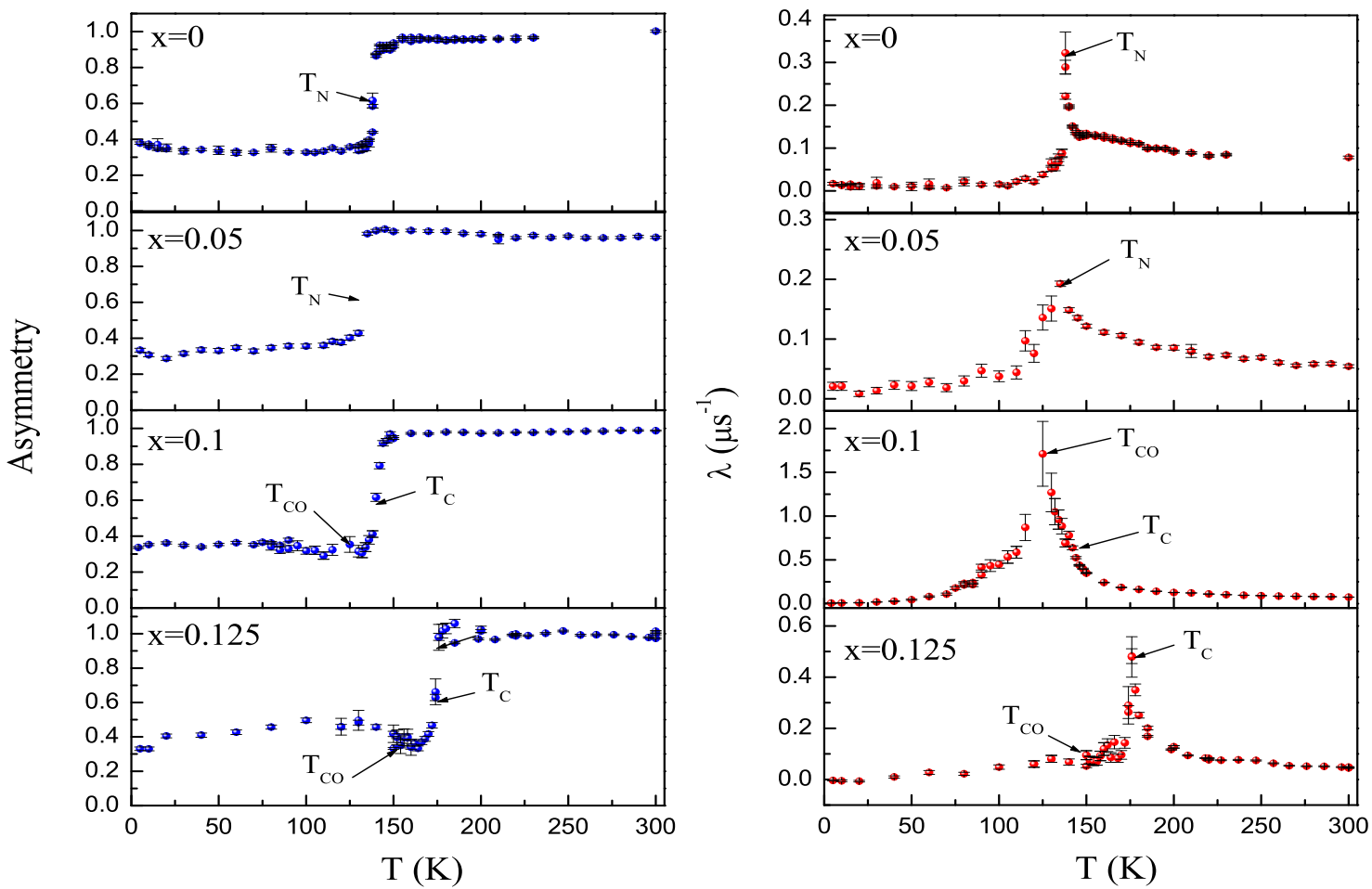


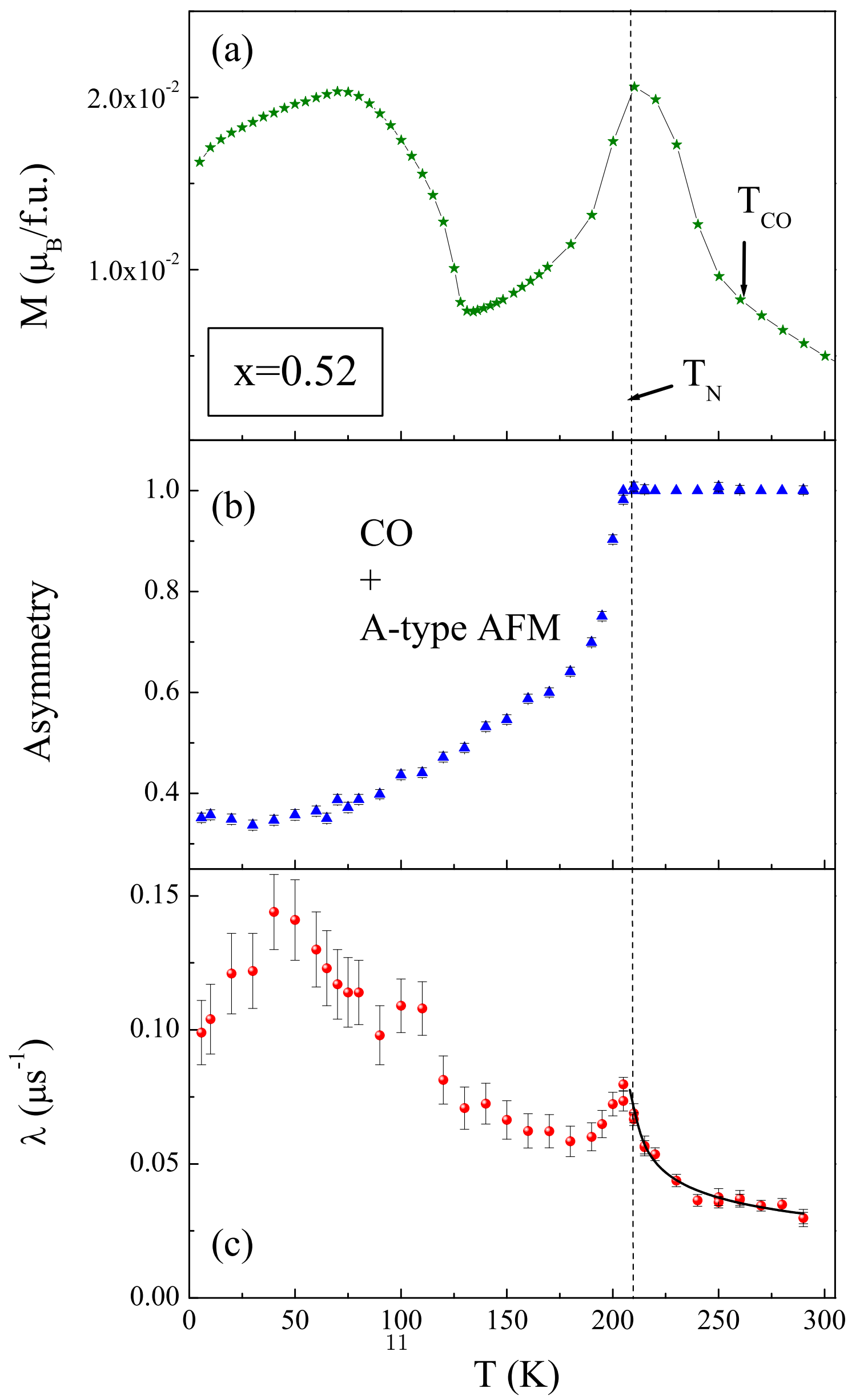

